\title{
Detecção de anticorpos IgG anti-Trypanosoma vivax em bovinos através do teste de Imunofluorescência indireta ${ }^{1}$
}

\author{
Neurisvan R. Guerra ${ }^{2}$, Maria F.M. Monteiro² ${ }^{2}$ Hévila M.M. Sandes², Nadine Louise \\ Nicolau da Cruz ${ }^{2}$, Carlos A.N. Ramos ${ }^{2}$, Vania Lúcia de Assis Santana ${ }^{3}$, Marcilia \\ Maria Alves de Souza ${ }^{3}$ e Leucio Câmara Alves ${ }^{2 *}$
}

\begin{abstract}
Guerra N.R., Monteiro M.F.M., Sandes H.M.M., Cruz N.L.N., Ramos C.A.N., Santana V.L.A., Souza M.M.A. \& Alves L.C. 2013. [Detection of IgG antibodies against Trypanosoma vivax in cattle by indirect immunofluorescence test.] Detecção de anticorpos IgG anti-Trypanosoma vivax em bovinos através do teste de Imunofluorescência indireta. Pesquisa Veterinária Brasileira 33(12):1423-1426. Laboratório de Doenças Parasitárias dos Animais Domésticos, Departamento de Medicina Veterinária, Universidade Federal Rural de Pernambuco, Rua Dom Manuel de Medeiros s/n, Dois Irmãos, Recife, PE 52171-900, Brazil. E-mail: leucioalves@gmail.com

Trypanosoma vivax infects a wide range of wild and domestic ungulates, causing important losses for the livestock industry. The aim of the present study was to assess the detection of IgG antibodies against T. vivax in cattle from the state of Pernambuco, Brazil. Therefore, we analyzed 2.053 blood serum samples from cattle herds of municipalities in Pernambuco, what was made by Immunofluorescence Assay. The overall seroprevalence of IgG antibodies against T. vivax in cattle was $13.93 \%$ (286/2053). The frequencies, by region, varied from $11.90 \%$ to $15.99 \%$. Thus, the data obtained allowed to characterize the state of Pernambuco as an area of enzootic instability for T. vivax. The frequency herein reported (i.e., 13.93\%) indicates that Pernambuco is an endemic area for T. vivax, this parasite being spread throughout the state.
\end{abstract}

INDEX TERMS: Trypanosoma vivax, protozoan, trypanosomiasis, cattle, serology, frequency, enzootic instability.

RESUMO.- Trypanosoma vivax infecta uma grande variedade de animais ungulados selvagens e domésticos, podendo causar grande impacto na produção de ruminantes. Este trabalho teve como objetivo avaliar a detecção de anticorpos IgG anti-Trypanosoma vivax em bovinos provenientes do estado de Pernambuco, Brasil. Para tanto, foram analisadas 2,053 amostras de soro sanguíneo de bovinos provenientes de rebanhos de municípios do estado de Pernambuco, os quais foram analisados através da Reação de Imunofluorescência Indireta. Das amostras testadas 13,93\% (286/2.053) foram reagentes para anticorpos IgG anti-Trypanosoma vi-

\footnotetext{
${ }^{1}$ Recebido em 18 de junho de 2013.

Aceito para publicação em 11 de outubro de 2013.

${ }^{2}$ Laboratório de Doenças Parasitárias dos Animais Domésticos, Departamento de Medicina Veterinária, Universidade Federal Rural de Pernambuco (UFRPE), Rua Dom Manuel de Medeiros s/n, Dois Irmãos, Recife, PE 52171-900, Brasil. *Autor para correspondência: leucioalves@gmail.com

${ }^{3}$ Laboratório Nacional Agropecuário (Lanagro), Rua D. Manoel de Medeiros s/n, Dois Irmãos, Recife, PE52171-120.
}

vax. As freqüências, por mesorregião, variaram de 11,90\% a $15,99 \%$. Assim, os dados obtidos permitiram a caracterização do estado de Pernambuco como uma área de instabilidade enzoótica e sugere que o estado Pernambuco é área endêmica para Trypanosoma vivax e este parasito está distribuído por todo o estado.

TERMOS DE INDEXAÇÃO: Trypanosoma vivax, protozoário, tripanossomíase, bovinos, sorologia, frequência, instabilidade enzoótica.

\section{INTRODUÇÃO}

O hematozoário Trypanosoma vivax infecta uma grande variedade de animais ungulados selvagens e domésticos, podendo causar grande impacto na produção de ruminantes na África, Américas Central e do Sul e Caribe (Osório et al. 2008).

Animais infectados podem apresentar-se assintomáticos, ou exibir sinais clínicos como anemia, perda de peso, sinais neurológicos, abortos (Batista et al. 2007), agalaxia, 
quedas naprodução de leite e carne, e, eventualmente, morte (Delafosse 2006).

No Brasil, T. vivax foi diagnosticado no Estado do Pará em 1972, infectando búfalo (Madruga et al. 1999). Em seguida, o protozoário foi detectado nos estados do Amapá (Serra-Freire 1981), Mato Grosso ( Osório et al. 2008), Tocantins (Linhares et al. 2006), Minas Gerais (Carvalho et al. 2008), Paraíba (Batista et al. 2007), Maranhão (Guerra et al. 2008) e recentemente no estado de Pernambuco (Pimentel et al. 2012).

Estudos sobre T. vivax no Brasil foram pontuais (Silva et al. 1996, Ventura et al. 2001, Cortez et al. 2006, Madruga et al. 2006, Baptista-Filho et al. 2011) em função da descrição da ocorrência ou prevalência da doença em um determinado local.

Tendo em vista a escassez de informações sobre a frequência de T. vivax em bovinos no Estado de Pernambuco, este trabalho tem como objetivo avaliar a detecção de anticorpos IgG anti-Trypanosoma vivax em bovinos provenientes do estado de Pernambuco, Brasil.

\section{MATERIAL E MÉTODOS}

O Estado de Pernambuco ( $8^{\circ} 4^{\prime} 14^{\prime \prime}$ S, 37 $15^{\prime} 57^{\prime \prime}$ ) $)$ está localizado na região Nordeste do Brasil. Possuindo uma área total de $98.938 \mathrm{~km}^{2}$ e uma população de 8.541 .250 habitantes (IBGE 2010).

Foram analisadas 2.053 amostras de soro sanguíneo de bovinos a partir do banco de soros gentilmente cedido pelo Laboratório Nacional Agropecuário em Pernambuco (Lanagro/ MAPA). Tais amostras eram provenientes de rebanhos de quatro mesorregiões do estado de Pernambuco a saber: Sertão, Agreste, Zona da Mata e Litoral. Os testes foram realizados através da Reação de Imunofluorescência Indireta (RIFI) segundo Silva et al. (2002).

\section{RESULTADOS}

Das 2.053 amostras testadas 13,93\% (286/2.053) foram reagentes para anticorpos IgG anti-Trypanosoma vivax. Este é o primeiro inquérito sorológico para detecção de anticorpos contra T. vivax em bovinos no estado de Pernambuco, os quais tiveram presentes em todas as mesorregiões estudadas (Quadro 1).

Esses dados indicam que as freqüências, por mesorregião, variaram de $11,90 \%$ a $15,99 \%$. No entanto, a análise estatística não revelou diferenças significativas entre frequências nas mesorregiões (Quadro 1) ( $p>0.05)\left(\chi^{2}=3,7\right.$; $\mathrm{p}=0,2962$ ).

Quadro 1. Fequência Absoluta (FA) e Frequência Relativa (FR) de bovinos reagentes ao teste de Imunofluorescência Indireta para pesquisa de anticorpos IgG anti-Trypanosoma vivax em diferentes mesorregiões do Estado de Pernambuco

\begin{tabular}{lccccc}
\hline Mesorregiões & \multicolumn{2}{c}{ Positivos } & & \multicolumn{2}{c}{ Negativos } \\
\cline { 2 - 3 } \cline { 6 - 6 } & FA & FR(\%) & & FA & FR(\%) \\
\hline Litoral & $32 / 269$ & 11,90 & & $237 / 269$ & 88,10 \\
Zona da Mata & $49 / 397$ & 12,34 & & $348 / 397$ & 87,66 \\
Agreste & $118 / 843$ & 14,00 & & $725 / 843$ & 86,00 \\
Sertão & $87 / 544$ & 15,99 & & $457 / 544$ & 84,01 \\
TOTAL & $286 / 2053$ & 13,93 & & $1767 / 2053$ & 86,07
\end{tabular}

\section{DISCUSSÃO}

Os resultados aqui foram superiores às taxas de prevalência encontradas em Uganda, Zambia, Guyana, Etiópia e Perú que variaram de $0,05 \%$ a $4,8 \%$ (Craig 1975, Magona et al. 1999, Quispe et al. 2003, Sinyangwe 2004, Mulaw 2011) e inferiores àquelas registradas por Toro (1976), Omotainse (1993) Abebe \& Jobre (1996), Abenga et al. (2004), Guedes Jr (2006), Ezebuiro (2009) e Suárez (2009) que relataram a presença de Trypanosoma vivax em $20,7 \%$ a $100 \%$ dos bovinos procedentes da Venezuela, Etiópia, Nigéria e Brasil.

A variação nas prevalências podem ser reflexo de diferentes formas de manejo, uso de anti-parasitários em algumas regiões além de fatores ligados aos vetores como sua distribuição e competência vetorial (Swai \& Kaaya 2012). Além disso, esses estudos foram realizados em diferentes estações do ano o que implica em taxas de infecção por T. vivax variadas pois sabe-se que a densidade dos vetores aumenta na dependência das estações climáticas (Onditi et al. 2007).

Outro ponto importante a ser considerado é a variação nas raças utilizadas nos diversos estudos devido ao fenômeno da tripanotolerância. Esse termo é utilizado para caracterizar algumas raças como N'Dama, Baoulé, Muturu, Dahoumey e Lagune (Osório et al. 2008) que são relativamente resistentes a infecção de T. vivax apresentando resistência natural e permanecem na condição de portadores assintomáticos (Mattioli \& Wilson 1996). Nesses animais, os títulos de anticorpos contra antígenos não variantes diferem entre animais resistentes e susceptíveis, observando-se altos títulos de IgG1 (Naessens et al. 2002).

Por outro lado, os resultados aqui obtidos estão próximos daqueles observados em regiões com clima e manejos semelhantes àqueles encontrados nas mesorregiões do presente estudo como relatado por Corten(1988), que obteve $10,22 \%$ de positividade em inquérito realizado na Zambia, e por Connor \& Halliwell (1987) que encontraram 17\% de prevalência de T. vivax, na Tanzânia.

Neste trabalho, os dados obtidos permitiram a caracterização do estado de Pernambuco como uma área de instabilidade enzoótica para T. vivax, de acordo com o conceito citado por Smith et al. (2000). Situação contrária ocorre na região do Pantanal, no Brasil, onde a presença de T. vivax raramente está associada com doença clínica em bovinos, búfalos e veados (Dávila et al. 2003).

Essa instabilidade enzoótica ocorre provavelmente em consequência do ambiente desfavorável para o desenvolvimento de vetores tais como tabanídeos (Paiva et al. 2000), Haematobia irritans e Stomoxys calcitrans (Cadiolli et al. 2012) durante a maior parte do ano em virtude da existência de períodos prolongados de secas e altas temperaturas (Batista et al. 2008).

É provável que a introdução desse parasito em Pernambuco tenha ocorrido devido ao trânsito de animais cronicamente infectados de regiões de ocorrência para regiões livres do protozoário (Pimentel et al. 2012).

Nos últimos anos, a fim de melhorar os rebanhos geneticamente, tem havido uma grande introdução de animais a partir de diferentes regiões do país no Nordeste. Este trânsito de animais pode ter contribuído para a entrada desse protozoário na região estudada (Batista et al. 2008). 
Razões semelhantes foram propostas por Carvalho et al. (2008), em Minas Gerais, onde um surto de T. vivax foi relatado. Este movimento de animais tem sido importante para a dispersão deste protozoário no Brasil (Linhares et al. 2006).

Assim, considerando o aumento da área endêmica de T. vivax no Brasil e a presença desse hemoprotozoário em vários países da América Latina (Dávila et al. 2003), provocando perdas econômicas importantes para a bovinocultura (Seidl et al. 1999), o diagnóstico epidemiológico e etiológico tornam-se imprescindíveis (Madruga et al. 2006).

É importante destacar que em nosso estudo boa parte dos animais testados são criados juntos com outras espécies, como cabras, ovelhas e búfalos, que são considerados potenciais reservatórios assintomáticos do parasita (Rodrigues et al. 2008).

Agradecimentos.- Ao Laboratório Nacional Agropecuário de Pernambuco (Lanagro), pelas amostras de soros sanguíneos de bovinos. À Fundação de Amparo à Ciência e Tecnologia do Estado de Pernambuco (Facepe), pela concessão de bolsa de mestrado.

\section{REFERÊNCIAS}

Abebe G. \& Jobre Y. 1996. Trypanosomiasis: a threat to cattle production in Ethiopia. Revta Med. Vet. 147:897-902.

Abenga J.N., Enwezor F.N.C., Lawani F.A.G., Osue H.O. \& Ikemereh E.C.D. 2004. Trypanosome prevalence in cattle in Lere Area in Kaduna State, North Central Nigeria. Revue Élev. Méd. Vét. Pays Trop. 57:45-48.

Baptista-Filho L.C.F., Fernandes A.C.C., Silva T.I.B., Souza A.C.M., Sandes H.M.M., Alves L.C. \& Melo L.E.H. 2011. Infecção por Trypanosoma vivax em bovinos leiteiros criados no estado de Pernambuco: relato de caso. Vet. Zootec.18:919-921.

Batista J.S., Bezerra F.S.B., Lira R.A., Carvalho J.R.G., Neto A.M.R., Petri A.A. \& Teixeira M.M.G. 2008. Aspectos clínicos, epidemiológicos e patológicos da infecção natural em bovinos por Trypanosoma vivax na Paraíba. Pesq. Vet. Bras. 28:63-69.

Batista J.S., Riet-Correa F., Teixeira M.M.G., Madruga C.R., Simões S.D.V. \& Maia T.F. 2007. Trypanosomiasis by Trypanosoma vivax in cattle in the Brazilian semiarid: description of an outbreak and lesions in the nervous system. Vet. Parasitol. 143:174-181.

Cadioli F.A., Barnabé P.A., Machado R.Z., Teixeira M.C.A., André M.R., Sampaio P.H., Fidélis Junior O.L., Teixeira M.M.G. \& Marques L.C. 2012. First report of Trypanosoma vivax outbreak in dairy cattle in São Paulo State, Brazil. Revta Bras. Parasitol. Vet. 21:118-124.

Carvalho A.U., Abrão D.C., Facury Filho E.J., Paes P.R.O. \& Ribeiro M.F.B. 2008. Ocorrência de Trypanosoma vivax no estado de Minas Gerais. Arq. Bras. Med. Vet. Zootec. 60:769-771.

Connor R.J. \& Hallwell R.W. 1987. Bovine trypanosomiasis in southern Tanzania: parasitological and serological survey of prevalence. Trop. Anim. Health Prod. 19:165-172.

Corten J.J.F.M., TerHuurne A.A.H.M., Moorhouse P.D.S. \& De Rooij R.C. 1988. Prevalence of trypanosomiasis in cattle in South-West Zambia. Trop. Anim. Health Prod. 20:78-84.

Cortez A.P., Ventura R.M., Rodrigues A.C., Batista J.S., Paiva F., Añez N., Machado R.Z., Gibson W.C. \& Teixeira M.M.G. 2006. The taxonomic and phylogenetic relationships of Trypanosoma vivax from South America and Africa. Parasitology 133:159-169.

Craig T.M. 1975. A prevalência de parasitos de bovinos em vários ambientes dentro do país várzea tropical da Guiana. PhD thesis Texas A \& M University, Austin, Texas.

Dávila A.M.R., Herrera H.M., Schlebinger T., Souza S.S. \& Traub-Cseko Y.M. 2003. Using PCR for unraveling the cryptic epizootiology of livestock trypanosomosis in the Pantanal, Brazil. Vet. Parasitol. 117:1-13.
Delafosse A., Thébaud E., Desquesnes M., Desquesnes M. \& Michaux Y. 2006. Epidemiology of Trypanosoma vivax infection in cattle in the tsetse free area of Lake Chad. Prev. Vet. Med. 74:108-119.

Ezebuiro O.G.C., Abenga J.N. \& Ekejindu G.O.C. 2009. The prevalence of trypanosome infection in trade cattle, goats and sheep slaughtered at the Kaduna abattoir. Afr. J. Clin. Exp. Microbiol. 10:15-25.

Guedes Junior D.S. 2006. Frequência de anticorpos para agentes da tristeza parasitária bovina, Trypanosoma vivax e Borrelia sp. em bovinos do Nordeste do Estado do Pará, Brasil. Dissertação de Mestrado, Instituto de Veterinária, Universidade Federal do Rio de Janeiro, Seropédica, RJ.

Guerra R.M.S., Feitosa Jr A.B., Santos H.P., Silva A.L.A. \& Santos C.G. 2008. Biometry of Trypanosoma vivax found in a calf in the state of Maranhão, Brazil. Ciência Rural 38:833-835.

IBGE 2010. Instituto Brasileiro de Geografia e Estatística. Disponível em <http://www.censo2010.ibge.gov.br/dados_divulgados/index.php? uf=26> Acesso em 27 nov. 2012.

Linhares G.F.C., Dias Filho F.D., Fernandes P.R. \& Duarte S.C. 2006. Tripanossomíase em bovinos no município de Formoso do Araguaia, Tocantins: relato de caso. Ciênc. Anim. Bras. 7:455-460.

Madruga C.R., Araújo F.R., Lima Júnior M.S.C. \& Melo E.S.P. 2006. Comparação de métodos de extração do DNA e avaliação de reações da polimerase em cadeia (PCR) para o diagnóstico de Trypanosoma (Dutonella) vivax. Circ. Téc. 34, Embrapa,Brasília, p.1-8.

Madruga C.R., Morzaria S. \& Majiwa P.O. 1999. Caracterização genética do Trypanosoma vivax isolado no pantanal do Estado de Mato Grosso e o diagnóstico diferencial da infecção por Trypanosoma evansi pela reação em cadeia da polimerase (PCR). Resultados preliminares. Pesquisa em Andamento 49, Embrapa Gado de Corte. 5p. Disponível em <http:// www.cnpgc.embrapa.br/publicacoes/pa/pa49.html> Acessado em 11 fev. 2013.

Magona J.W., Kakaine D.W. \& Mayende J.S.P. 1999. Prevalence and distribution of animal trypanosomosis on Buvuma islands in Lake Victoria, Uganda. Trop. Anim. Health Prod. 31:83-87.

Mattioli R.C. \& Wilson R.T. 1996. Trypanosomes, tsetse and trypanotolerance: coevolution in Tropical Africa. Parassitol, 38:531-535.

Mulaw S., Addis M. \& Froms A. 2011. Study on the prevalence of major trypanosomes affecting bovine in tsetse infested Asosa District of BenishangulGumuz Regional State, Western Ethiopia. Global Vet. 7:330-336.

Naessens J., Teale A.J. \& Sileghem M. 2002. Identification of mechanisms of natural resistance to African trypanosomiasis in cattle. Vet. Immunol. Immunopathol. 87:187-194.

Omotainse S.O., Edeghere H., Omoogum G.A., Elhassan E.O., Thompson G., Igweh C.A., Ukah J.A.C., Ikenga M.A. \& Halid I. 1993. The Prevalence of animal trypanosomosis In Konshishalocal Government area of Benue State, Nigeria. Israel J. Vet. Med. 55:210-220.

Onditi S.J., Silayo R.S., Kimera S.I., Kimbita E.N. \& Mbilu T.J.N.K. 2007. Preliminary studies on prevalence and importance of goat trypanosomosis in selected farms in Morogoro District, Tanzania. Livest. Res. Rural Dev. 19(5). <http://www.lrrd.org/lrrd19/5/ondi19065.htm> Acessado em 10 jun. 2013.

Osório A.L.A.R., Madruga C.R., Desquenes M., Soares C.O., Ribeiro L.R.R. \& Da Costa C.G. 2008. Trypanosoma (Dutonella) vivax: its biology, epidemiology, pathogenesis, and introduction in the New World: a review. Mem. Inst. Oswaldo Cruz 103:1-13.

Paiva F., Lemos R.A.A., Nakasato L., Mori A.E., Brum K.B. \& Bernardo K.C. 2000. Trypanosoma vivax em bovinos no pantanal do estado do Mato Grosso do Sul, Brasil. I. Acompanhamento clínico, laboratorial e anatomopatológico de rebanhos infectados. Revta Bras. Parasitol. Vet. 9:135141.

Pimentel D.S., Ramos C.A.N., Ramos R.A.N., Araújo F.R., Borba M.L., Faustino M.A.G. \& Alves L.C. 2012. First report and molecular characterization of Trypanosoma vivax in cattle from state of Pernambuco, Brazil. Vet. Parasitol. 185:286-289.

Quispe A.P., Chávez V.A., Casas A.E., Trigueros V.A. \& Suárez A.F. 2003. Prevalencia de Trypanosoma vivax en bovinos de la provincia de Coronel Portillo, Ucayali. Revta Invest. Vet. Peru 14:161-165. 
Rodrigues A.C., Neves L., Garcia H.A.,Viola L.B., Marcili A., Silva F.M., Sigauque I., Batista J.S., Paiva F. \& Teixeira M.M.G. 2008. Phylogenetic analysis of Trypanosoma vivax supports the separation of South American/West African from East African isolates and a new T. vivax-like genotype infecting a Nyala antelope from Mozambique. Parasitol. 135:1317-1328.

Seidl A., Dávila A.M.R. \& Silva R.A.M.S. 1999. Estimated financial impact of Trypanosoma vivax on the Brazilian Pantanal and Bolivian lowlands. Mem. Inst. Oswaldo Cruz 94:269-272.

Serra-Freire N.M. 1981. Oiapoque: outro foco de Trypanosoma vivax no Brasil. Revta Bras. Med. Vet. 4:30-31.

Silva R.A.M.S., Seidl A., Ramirez L. \& Dávila A.M.R. 2002. Tripanosoma evansi e Trypanosoma vivax: biologia, diagnóstico e controle. Embrapa Pantanal, Corumbá, MS. 141p. <http://www.cpap.embrapa.br/publicacoes/online/Livro015.pdf> Acessado em 11 fev. 2013.

Silva R.A.M.S., Silva J.A., Schneider R.C., Freitas J., Mesquita D., Mesquita T., Ramirez L., Dávila A.M.R. \& Pereira M.E.B. 1996. Outbreak of trypanosomiasis due to Trypanosoma vivax (Ziemann, 1905) in bovines of the Pantanal, Brazil. Mem. Inst. Oswaldo Cruz 91:561-562.
Sinyangwe L., Delespaux V., Brandt J., Geerts S., Mubanga J., Machila N., Holmes P.H. \& Eisler M.C. 2004. Trypanocidal drug resistance in eastern province of Zambia. Vet. Parasitol. 119:125-135.

Smith R.D., Evans D.E., Martins J.R., Ceresér V.H., Correa B.L., Petraccia C., Cardozo H., Solari M.A. \& Nari A. 2000. Babesiosis (Babesia bovis) stability in unstable environments. Annals N.Y. Acad. Sci. 916:510-520.

Suárez C., García F., Román D., Coronado A., Perrone T., Reyna A. \& Parra N. 2009. Factores de riesgo asociados a la tripanosomosis bovina en explotaciones ganaderas de Venezuela. Zootec. Trop. 27:363-372.

Swai E.S. \& Kaaya J.E. 2012. A parasitological survey for bovine trypanosomosis in the livestock/wildlife ecozone of Northern Tanzania. Vet. World 5:459-464.

Toro E.M. 1976. Diagnostico serológico de la tripanosomiasis bovina usando una prueba de aglutinación capilar. Vet. Trop. 1:15-40.

Ventura R.M., Paiva F., Silva R.A.M.S., Takeda G.F., Buck G.A. \& Teixeira M.M.G. 2001. Trypanosoma vivax: characterization of the spliced-leader gene for a Brazilian stock and species-specific detection by PCR amplification of an intergenic space sequence. Exp. Parasitol. 99:37-48. 